\title{
LETTER
}

\section{Elasticity analysis in epidemiology: an application to tick-borne infections}

\author{
Amy Matser, ${ }^{1 \dagger}$ Nienke \\ Hartemink, ${ }^{1 *,+}$ Hans \\ Heesterbeek, ${ }^{1}$ Alison Galvani ${ }^{2}$ \\ and Stephen Davis ${ }^{2}$ \\ ${ }^{1}$ Theoretical Epidemiology, \\ Yalelaan 7, University of \\ Utrecht, Utrecht, The \\ Netherlands \\ ${ }^{2}$ School of Public Health, 60 \\ College Street, Yale University, \\ New Haven, USA \\ *Correspondence: \\ E-mail: n.a.hartemink@uu.nl
}

\begin{abstract}
The application of projection matrices in population biology to plant and animal populations has a parallel in infectious disease ecology when next-generation matrices (NGMs) are used to characterize growth in numbers of infected hosts $\left(R_{0}\right)$. The NGM is appropriate for multi-host pathogens, where each matrix element represents the number of cases of one type of host arising from a single infected individual of another type. For projection matrices, calculations of the sensitivity and elasticity of the population growth rate to changes in the matrix elements has generated insight into plant and animal populations. These same perturbation analyses can be used for infectious disease systems. To illustrate this in detail we parameterized an NGM for seven tick-borne zoonoses and compared them in terms of the contributions to $R_{0}$ from three different routes of transmission between ticks, and between ticks and vertebrate hosts. The definition of host type may be the species of the host or the route of infection, or, as was the case for the set of tick-borne pathogens, a combination of species and the life stage at infection. This freedom means that there is a broad range of disease systems and questions for which the methodology is appropriate.
\end{abstract}

\section{Keywords}

Anaplasma phagocytophila, Borrelia burgdorferi, Crimean-Congo haemorrhagic fever virus, elasticities, Kyasanur forest disease virus, next-generation matrix, Rickettsia rickettsii, Thogoto virus, tick-borne encephalitis virus.

Ecology Letters (2009) 12: 1298-1305

\section{INTRODUCTION}

Projection matrices in population biology relate life-history events acting on individual animals and plants to population-level growth rates. Over the last two decades, the interpretation of elasticities of projection matrices as relative contributions to the population growth rate $(\lambda)$ has established elasticity analysis as a standard tool in population biology (Caswell 2001). The insights provided by these analyses in the fields of conservation, biocontrol, sustainability and evolutionary ecology have been varied and substantial (Silvertown et al. 1996; Benton \& Grant 1999). For example, perturbation analysis has facilitated the identification of those life stages that should be targeted to most effectively promote species conservation (Benton \& Grant 1999).

\footnotetext{
${ }^{\dagger}$ Authors Amy Matser and Nienke Hartemink contributed equally to this work.
}

In epidemiology, the spread of infection is typically characterized by the basic reproduction number, $R_{0}$. This quantity is fundamental to the theory and management of infectious diseases. In the simplest of settings, when there is a homogeneous and well-mixed population of hosts, $R_{0}$ is defined as the expected number of secondary cases produced by a single primary case in a wholly susceptible population. To handle pathogens that infect multiple hosts, Diekmann et al. (1990) introduced a next-generation matrix (NGM), the dimension of which is determined by the number of epidemiologically distinct, infected and infectious host types and whose entries are all host type-specific reproduction numbers [see also Diekmann \& Heesterbeek (2000)]. That is, each of the matrix elements $k_{i j}$ is the expected number of infected of type $i$ produced by a single infectious individual of type $j$. The leading eigenvalue of the NGM is $R_{0}$. Here begins an intriguing parallel with projection matrices as applied to study plant and animal populations (Fig. 1). 


\section{Projection matrices in population ecology}

The life cycle graph represents transitions between demographic stage-classes (age or size) over a fixed time-step. Arcs represent transitions of individuals between the stage-classes or reproduction. A wellknown example is the life cycle graph for killer whales (Orcinus orca) ${ }^{1}$

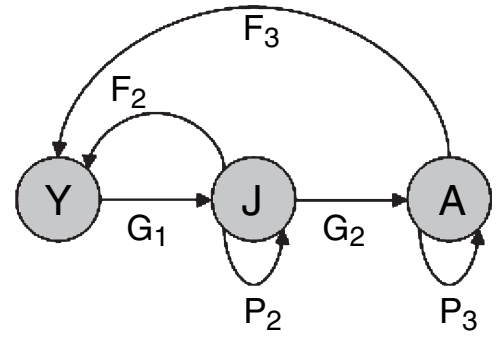

$Y=$ yearling

$\mathrm{J}=$ juvenile

$A=$ adult

The projection matrix, $A$, describes how the size and composition of the current population, $n_{t}$ determines the future population, $n_{t+1}$.

$$
n_{t+1}=A n_{t}
$$

The entries of the projection matrix describe the transitions from one stage to another, e.g. $G_{1}$ is the transition rate from yearlings into juveniles and $F_{2}$ and $F_{3}$ are the fecundity rates of juveniles and adults, respectively.

$$
A=\left[\begin{array}{ccc}
0 & F_{2} & F_{3} \\
G_{1} & P_{2} & 0 \\
0 & G_{2} & P_{3}
\end{array}\right]
$$

The growth rate $\lambda$ of the population is given by the dominant eigenvalue of $A$.

\section{Next-generation matrices (NGM) in infectious disease ecology}

A next-generation matrix describes the increase in infecteds over generations. Its graphical representation consists of nodes representing the different types-at-infection and arcs representing reproduction numbers, i.e. the expected numbers of infecteds of a certain type produced by another type. An example is tick-borne encephalitis virus in Ixodes ricinus ${ }^{2}$.

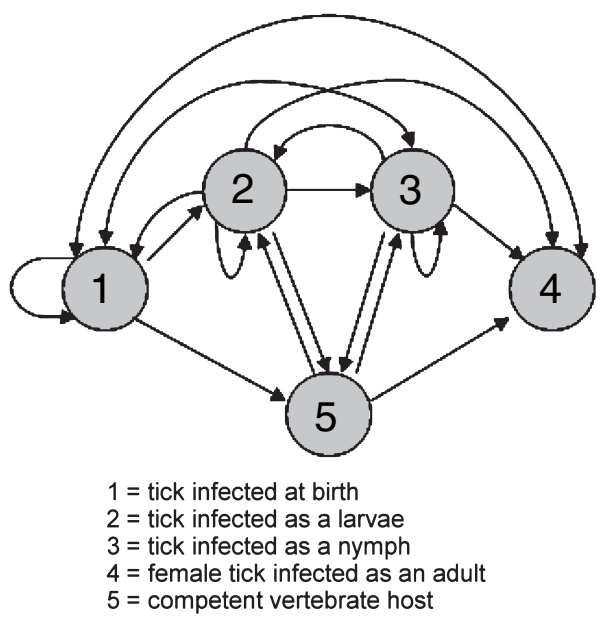

The next-generation matrix $K$ describes changes in composition and number of infected individuals over generations.

$$
\phi_{n+1}=K \phi_{n}
$$

The entries of $K$ describe the transmission process between all pairs of types, e.g. entry $k_{12}$ represents the number of ticks infected at birth produced by a tick that was infected while feeding as a larva.

$K=\left[\begin{array}{lllcc}k_{11} & k_{12} & k_{13} & k_{14} & 0 \\ k_{21} & k_{22} & k_{23} & 0 & k_{25} \\ k_{31} & k_{32} & k_{33} & 0 & k_{35} \\ k_{41} & k_{42} & k_{43} & 0 & k_{45} \\ k_{51} & k_{52} & k_{53} & 0 & 0\end{array}\right]$

The generation growth rate (basic reproduction number) $R_{0}$ is given by the dominant eigenvalue of $K$

Figure 1 Summary of the parallel between projection matrices for plants and animals and next-generation matrices for pathogens. ${ }^{1}$ indicates Brault \& Caswell (1993); ${ }^{2}$ indicates Hartemink et al. (2008).

Multi-host pathogens represent the majority of infectious disease systems (Woolhouse et al. 2001), including that group of pathogens that are zoonoses of major public health concern. Key examples include rabies, sleeping sickness, Lyme disease and influenza viruses such as the H1N1 strain that recently emerged as a relatively benign form of pandemic flu. The NGM methodology is hence an important step in moving away from the easier paradigm of transmission among a population consisting of a single host species, and towards the more commonly encountered multi-host pathogen circulating within and between populations of co-existing hosts. The methodology allows calculation of $R_{0}$ for multi-host pathogens where the threshold value of 1 is retained and the interpretation of the numerical value is consistent with that in the simpler setting of a single host, and therefore meaningful. It is also worth noting here that if a particular host type (e.g. humans) is of principal interest then a shift in perspective towards this host, using the type reproduction number (Roberts \& Heesterbeek, 2003), is easily achieved. This means that an 
NGM can provide a scientific basis for the proportion of $a$ particular host type that needs to be vaccinated to eliminate an infection. The additional benefit of NGMs, of being able to carry over the perturbation analyses worked out in the context of projection matrices, has largely gone unexplored. We rectify this here, principally by illustrating perturbation analysis in disease ecology using a subset of tick-borne pathogens, but also by emphasizing the broader relevance to complex, multi-host disease systems.

We first explain aspects of the methodology in detail, particularly as they need to be understood in the context of infectious disease systems. We then parameterize an NGM for seven zoonotic infections transmitted by hard ticks and explore the eco-epidemiological differences between these infections using composite elasticities (see section on 'Sensitivities, elasticities and composite elasticities'). We chose to use tick-borne pathogens as an example for several reasons. First, tick-borne diseases constitute a serious and persistent problem in many areas of the world (Randolph 2000; Steere 2001). Even though humans are often accidental hosts, playing no role in the spread of the pathogen, severe disease can develop from a single infectious tick bite. For this reason, tick-borne pathogens receive considerable attention, both in the laboratory and the field, which has resulted in a large body of literature (from which we were able to parameterize the NGMs). Second, even though there are important differences in the ecology, there is one important feature that these seven pathogens have in common: all are transmitted by hard ticks with comparable life cycles consisting of three post-egg stages. This enabled us to use the same NGM (presented by Hartemink et al. (2008)) for all seven pathogens. Third, the ecology of these pathogens is complex, with the potential for three different transmission routes. Transmission can occur when an infected tick causes a systemic infection in a vertebrate host, which may then pass the infection to susceptible ticks feeding on the host at a later time; this is referred to as systemic transmission. Tick-borne pathogens may also be transmitted from female adult tick to her offspring (transovarial transmission). Finally, a susceptible tick that feeds close to an infected tick on the same host can become infected without a systemic infection occurring in the host. This is known as non-systemic transmission. This complexity provides an ideal opportunity to illustrate the full potential of perturbation analyses for NGMs.

\section{PROJECTION MATRICES AND NGMS}

The key difference between a projection matrix and an NGM is that the former consists of transition probabilities and reproduction numbers calculated over the chosen time step (often 1 year) whereas the latter consists entirely of reproduction numbers, with no reference to the time period over which new infected individuals arise (Fig. 1). That is, just as the value of $R_{0}$ indicates nothing about the speed with which an epidemic might develop in real time, nor do the individual elements of an NGM. All elements of an NGM represent the expected number of new cases of a particular host type a second host type caused by over the entire infectious period. It is important to understand though that the infectious period of one host type might be a matter of weeks whereas a second host type might be infectious for years. A good example of this is the West African form of sleeping sickness, a chronic disease of humans caused by Trypanosoma brucei gambiense and transmitted by tsetse flies (Glossina spp.). In disease systems like this, many generations of infected can co-exist at the same point in time. An NGM cannot be used to calculate the total number of infected at any point in time.

The graphical representation of a projection matrix is also the life-cycle graph of the species under study. The graphical representation of an NGM (Fig. 1), which we refer to as the transmission graph from here onwards, does not describe the paths taken by individuals but is nevertheless a useful visualization of the host types (the nodes) and the possible transmission routes between them (the directed arcs). In the transmission graph shown in Fig. 1, four of the host types are ticks infected at different stages of their life cycle. It is crucial to understand that, in general, ticks are infected for life so that a tick infected as a larva may become a nymph and then an adult tick, but it never stops being a tick that was infected during the blood meal it took as a larva. That is, the host type is a property of an individual that does not change over time. If the host type is defined in terms of species alone then this will certainly be true, but more generally it is an important 'rule' when defining a set of host types. A host type should also be a group of hosts that are not only capable of being infected but they can themselves be infectious. Dead-end (accidental) hosts, such as humans for rabies, for example, are the equivalent of post-reproduction age or sizeclasses in population biology - they do not contribute to the population growth and are therefore set aside.

\section{SENSITIVITIES, ELASTICITIES AND COMPOSITE ELASTICITIES}

Elasticity analysis is a form of perturbation analysis, wherein one calculates the proportional response in a dependent variable resulting from a proportional perturbation in an independent variable. Sensitivity refers to the equivalent absolute change (Caswell 2001). Both are instructive to calculate, but in the context of projection matrices, elasticity analysis has been seen to be the fairer means of comparison, because the set of independent variables are measured on different scales (transition rates are constrained to lie between 0 and 1 , whereas measures of fecundity are not) 
and proportional perturbations therefore facilitate comparison in relative terms (Van Groenendael et al. 1988).

The key property of the elasticities of the dominant eigenvalue of a matrix to the matrix elements (for convenience we refer to these as element elasticities) is that they always sum to 1 (Caswell 2001). For projection matrices, this has led to their interpretation as the relative contribution to population growth from the respective transition in the life cycle (de Kroon et al. 1986). In the same sense, the element elasticities of an NGM can be interpreted as contributions to $R_{0}$. If a matrix entry is 0 , the corresponding element elasticity is 0 . Also, one can think of, for example, a $1 \%$ increase in $R_{0}$ as coming from a simultaneous $1 \%$ increase in all of the elements of the NGM, but the increase in some elements contributes more to the increase in $R_{0}$ than others. The relative contributions are given exactly by the elasticities.

Composite elasticities, sums of particular sets of element elasticities, follow from the interpretation of element elasticities as contributions to population growth rate or $R_{0}$. In population biology, composite elasticities are sums of the element elasticities that represent the same type of demographic transition. For plants, for example, composite elasticities have been used extensively to quantify the contribution of fecundity, growth and survival to the population growth rate. This becomes particularly interesting for comparative studies when sufficient demographic data is available to calculate element elasticities (and therefore composite elasticities too) for many species or for many populations. These have generally proven to be insightful for plants and animals (Brault \& Caswell 1993; Silvertown et al. 1996). In epidemiology, only Hartemink et al. (2008) have compared pathogens on the basis of composite elasticities, and in that study just two pathogens were compared. The results for seven tick-borne pathogens, using the same set of composite elasticities, are shown in Figs 2 and 3 (see section on 'Application to tick-borne pathogens' for details).

There is no restriction on which element elasticities should be grouped to form composite elasticities, and provided the rules mentioned in the preceding section are followed there is no restriction on how host types are defined. This is an important contrast to make with stage-structured population models (projection matrices) for plants and animals. There is some flexibility in the number of age- or stage-classes chosen, which is tied to the chosen time step of the model, but the division of the population always represents the progression of individuals. In an NGM, the choice of host types should be tailored to the specific disease system, and the definition of host type can be based on something obvious like host species (useful for a true multi-host pathogen like rabies), or something less intuitive such as the life stage of the insect vector that caused the infection (which could be useful for vector-borne diseases), or even the geographical location of
Table 1 Average $R_{0}$ values and the 95\% range of $R_{0}$ values from Latin Hypercube sampling

\begin{tabular}{ll}
\hline & $\begin{array}{l}\text { Average } R_{0} \\
(95 \% \text { range })\end{array}$ \\
\hline $\begin{array}{l}\text { Crimean-Congo heamorrhagic } \\
\text { fever virus }\end{array}$ & $2.18(1.31-4.64)$ \\
Rickettsia rickettsii & $8.24(3.54-14.58)$ \\
Kyasanur forest disease virus & $1.47(0.30-3.92)$ \\
Borrelia burgdorferi & $8.25(1.93-21.18)$ \\
Tick-borne encephalitis virus & $1.58(0.73-2.90)$ \\
Anaplasma phagocytophila & $3.90(0.74-10.76)$ \\
Thogoto virus & $6.01(1.56-13.51)$ \\
\hline
\end{tabular}

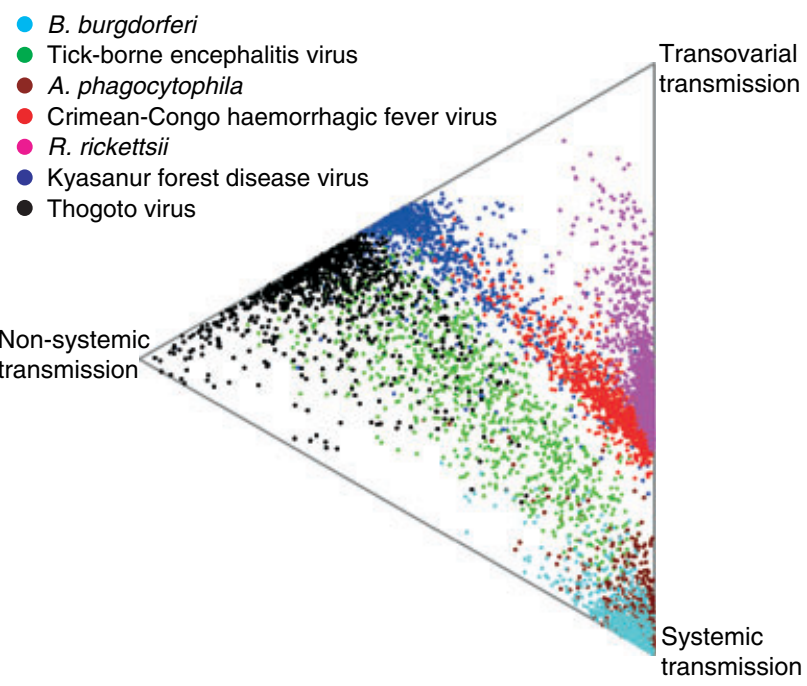

Figure 2 Balance of contributions to $R_{0}$ from the three transmission routes - systemic, transovarial and non-systemic - commonly available to tick-borne infections, as measured by composite elasticities taken from next-generation matrices (NGMs). One thousand points for each of the seven diseases were considered and were obtained by considering a Latin Hypercube sample from ranges set for the 31 biological parameters that enter the NGM. For the resulting matrices (1000 per disease), the elasticity of each of the matrix elements is calculated as: $e_{i j}=k_{i j} / \mathrm{R}_{0} \times \delta \mathrm{R}_{0} / \delta \mathrm{k}_{i j}$. As each element corresponds to only one type of disease transmission, the contributions of the three transmission routes can be assessed by summing the matrix elasticities that refer to the same transmission routes. The relative contribution of transovarial transmission to $R_{0}$, denoted as $C_{\text {to }}$ is then the sum of $e_{11}, \mathrm{e}_{12}, e_{13}$ and $e_{14}$. The contribution of systemic transmission $\left(C_{\mathrm{s}}\right)$ is the sum of the elements in row 5 and column 5 : $e_{51}, e_{52}, e_{53}, e_{25}, e_{35}$ and $e_{45}$. The remaining non-zero elements add up to the contribution of non-systemic transmission $\left(C_{\mathrm{ns}}\right): e_{21}, e_{22}, e_{23}, e_{31}, e_{32}, e_{33}, e_{41}, e_{42}$ and $e_{43}$. These three contributions must sum to 1 ; so for each of the 1000 points, the three contributions can be plotted in a ternary plot (also known as a De Finetti diagram in population genetics), where $X=\sqrt{3} \times\left(1-C_{\mathrm{ns}}\right)$ and $Y=1-\left(C_{\mathrm{s}}+C_{\mathrm{to}}\right)$. 


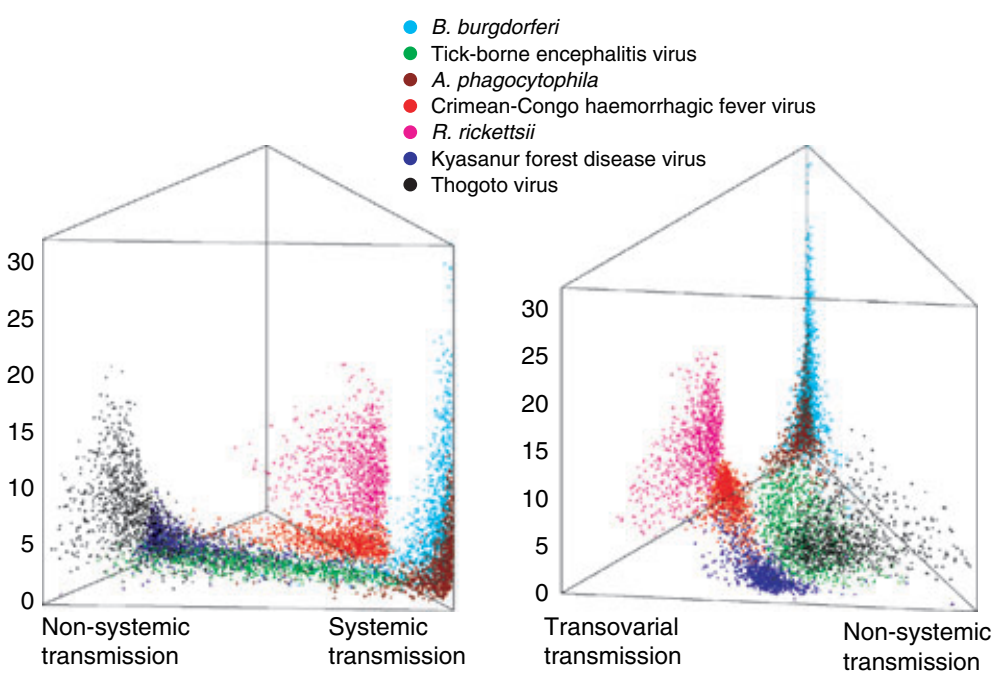

Figure 3 Two perspectives on the threedimensional ternary plot, showing the contributions to $R_{0}$ from the three transmission routes; on the vertical axis is the value of $R_{0}$. the host. A mix of such host type definitions is equally possible. There is considerable scope then for how composite elasticities might be defined to provide new perspectives on a disease system of interest. The results presented in Figs 2 and 3 , and the set of host types used to obtain them, are interesting in their own right for tick-borne disease specialists, but are by no means a template for work on other groups of pathogens. Rather, the purpose of these figures is principally to illustrate how element elasticities can be combined in an interesting way and how comparative studies can be based on them.

\section{APPLICATION TO TICK-BORNE PATHOGENS}

We parameterize the NGM proposed by Hartemink et al. (2008) for seven tick-borne pathogens and apply perturbation analyses to study the variation in a set of three composite elasticities, interpreted as contributions to $R_{0}$ from the three transmission routes observed. The pathogens are Borrelia burgdorferi (causing Lyme disease), Rickettsia rickettsii (Rocky Mountain spotted fever), Anaplasma phagocytophila (anaplasmosis), tick-borne encephalitis virus (TBEV), Crimean-Congo haemorrhagic fever virus (CCHFV), Thogoto virus and Kyasanur forest disease virus (KFDV). In Europe, Ixodes ricinus is one of the most important tick species involved in pathogen transmission; it transmits Borrelia burgdorferi, TBEV and A. phagocytophila. Rickettsia rickettsii is transmitted by Dermacentor spp. in the United States and KFDV is found in a small part of India and is transmitted by Haemaphysalis spinigera. The most important species involved in transmitting $\mathrm{CCHFV}$ is Hyalomma spp., found in Africa, Asia and parts of Europe.

The matrix model used to generate the results shown in Table 1, and Figs 2 and 3, as well as explanations for the many parameters involved and the definitions of the composite elasticities are presented in detail in the Supporting Information and the base model is the same as used by Hartemink et al. (2008). However, unlike Hartemink et al. (2008), the literature was used to set minimum and maximum values for the tick-/host-related biological parameters (Table S1) and the pathogen-specific parameters (Table S2) rather than trying to arrive at point estimates. This enables us to take into account the uncertainty surrounding the ecology of the tick species involved, the pathogens and their epidemiology and transmission routes. For each of the seven diseases, we created 1000 different parameterizations of the NGM by drawing samples from the parameter space defined by the ranges determined for each parameter. Samples were drawn using Latin Hypercube sampling techniques (Sanchez \& Blower 1997). Each sample produces an NGM, and hence an $R_{0}$ value, as well as composite elasticities representing contributions for systemic, non-systemic and transovarial transmission.

We plotted the composite elasticities for the three transmission routes on a ternary plot (Fig. 2) in the same way as composite elasticities representing contributions from growth, survival and fecundity have been for plant populations (Silvertown et al. 1996). The position on the triangle represents the balance of the three contributions (composite elasticities) to $R_{0}$ from systemic, transovarial and non-systemic transmission. Points on or very close to the three corners represent disease systems for which only one transmission route contributes to $R_{0}$. Points on or very near the edges indicate that only two of the three routes have significant contributions. Points appearing near the centre of the triangle represent almost equal contributions from all the three routes. As shown in Fig. 2, for B. burgdorferi and A. phagocytophila (both transmitted by $I$. ricinus) the largest contribution to $R_{0}$ is from systemic transmission. For TBEV (the third pathogen transmitted by $I$. ricinus), approximately half of the samples also have the largest contribution from systemic transmission, but for this pathogen, non-systemic transmission is very important too. KFDV and Thogoto 
virus have strong contributions from both non-systemic and transovarial transmissions. CCHFV and $R$. rickettsii rely on a combination of transovarial and systemic transmission.

In parameterizing the model of Hartemink et al. (2008), we identified gaps in the knowledge of tick-borne pathogens where either laboratory studies or field studies are weak or non-existent. Estimates of stage-specific tick survival for example, to which the results of the matrix model are known to be locally sensitive (Hartemink et al. 2008), proved difficult to find in the literature. Information on the efficiency of nonsystemic transmission between various pairings of the larval, nymph and adult stages was also absent, and the same parameter range was used for all pairings. The degree of uncertainty expressed in the $95 \%$ intervals for $R_{0}$ (Table 1 ) is a direct reflection of the general paucity of data. Nonetheless, the scatter for at least six of the sets of points in the ternary plot (Fig. 2) was relatively small, facilitating interesting comparisons between pathogens. The scatter of points for the seventh pathogen, TBEV, is relatively wide, receiving the largest contributions from either systemic or non-systemic transmission. The implication is that the uncertainty about the contributions of the transmission routes for this pathogen is larger than for the other six pathogens.

The corner of the triangle representing transovarial transmission appears to be relatively unoccupied. This may be because we have chosen seven zoonoses, and it is unlikely for any zoonotic disease to have a strong transmission capacity from only transovarial transmission as capacity for horizontal transmission is essentially a requirement to be a zoonosis. It is possible that we overestimated the contribution of transovarial transmission for $R$. rickettsii as mortality among infected ticks is higher than for uninfected ticks, and fecundity too is decreased by infection (Burgdorfer \& Varma 1967; Burgdorfer \& Brinton 1975). A third mechanism reducing the importance of transovarial transmission of $R$. rickettsii is vertical transmission interference; ticks co-infected with other Rickettsiae species do not transmit $R$. rickettsii to their progeny (Macaluso et al. 2001).

A third axis can be added to the ternary plot (Fig. 2) to show the values of $R_{0}$ associated with each of the points (Fig. 3 shows two perspectives of this three-dimensional plot). The range of values taken by $R_{0}$ across the seven diseases varies from below 1 to $c$. 30. The average $R_{0}$ value for each pathogen and the range within which $95 \%$ of the $R_{0}$ values calculated fell (there is one $R_{0}$ value for each of the 1000 Latin Hypercube samples) are shown in Table 1 . The vast majority of $R_{0}$ values are below 10. Pathogens with lower values of $R_{0}$ and many sample points with values below 1 (i.e. those for which the 95\% range includes 1) would be predicted to have more problems in being established in new areas. In TBEV, this has been linked to the 'spotted' distribution in Eurasia. We would also note that the KFDV, with a similar distribution for $R_{0}$ as TBEV, appears to be confined to a very small part of India.
The potential for high $R_{0}$ values can be generally associated with points closer to the corners, or edges, of the ternary plot, as shown in Fig. 3. A set of scatter plots of $R_{0}$ and the contributions from the three transmission routes (see Appendix S1 in Supporting Information) shows that this pattern is present most clearly in B. burgdorferi and A. phagocytophila. For TBEV, the points representing a high contribution from non-systemic transmission also happen to be the points with higher $R_{0}$ values. This result is consistent with the observations made by Randolph et al. (1999) that non-systemic transmission is the most efficient route of transmission for this pathogen. However, note that we do not go so far as to call these patterns trends or correlations. In the evaluation of the contribution of the different transmission routes to $R_{0}$, it is important to note that the 1000 points for each pathogen do not represent 1000 real disease systems, but random samples of parameter space. In particular, the variation in the scatter of points reflects uncertainty from field and laboratory data and not natural variation. With this in mind, the figures in the Supporting Information (see Figs S1 and S2 in Appendix S1) which show the orientation of the scatter of points and hence associations between $R_{0}$ and the balance of contributions from the transmission routes, should be treated purely as model output. For example, the scatters of B. burgdorferi, $A$. phagocytophila and $R$. rickettsii show the existence of a positive association between higher $R_{0}$ values and a higher contribution of systemic transmission but the model does not include any evolutionary or epidemiological constraints on the pathogen or hosts. This means that the positive associations here could entirely be a feature of the next-generation model, and in nature the opposite association could still be true. This is not to say that the model behaviour is in itself uninteresting, but that field data from distinct populations of hosts is required to draw real conclusions.

\section{DISCUSSION}

The application of perturbation analyses to projection matrices, familiar to many population biologists, has a parallel in the field of infectious disease epidemiology with respect to NGMs. We illustrated this parallel by describing the variation in a group of seven tick-borne pathogens, in terms of $R_{0}$ and in terms of the balance of contributions to $R_{0}$ from systemic, transovarial and non-systemic routes of transmission. The same approach may be used to describe variation between strains of the same aetiological agent or, as $R_{0}$ is just as much a property of the host population as the pathogen, variation between populations of hosts. If enough distinct geographical variants of a pathogen can be represented, then correlations among the elasticities or composite elasticities, or between the elasticities and $R_{0}$, or even the absence of correlation, is very likely to be a step forward in understanding the eco-epidemiology of the pathogen. 
Visualizing the contributions of the transmission routes to $R_{0}$ gives a novel perspective on the seven tick-borne pathogens and, as far as they are representative, the family of tick-borne pathogens. With the exception of B. burgdorferi and A. phagocytophila, the sets of points for the seven pathogens occupy distinct areas of the ternary plot. This, at the very least, implies diversity in the contributions of the different transmission routes to $R_{0}$. As the seven pathogens represent the state of an evolutionary process (albeit an ongoing one) the results tentatively imply that for tick-borne pathogens there are fitness benefits in maintaining multiple transmission routes. Somewhat contradicting this though is the general association in the model output (see Fig. S1) between the highest $R_{0}$ values and specialization in a single transmission route because $R_{0}$ values are often used as a measure of fitness for pathogens; see, for example, Gandon et al. (2001). Overall, the results for the seven pathogens are intriguing from an evolutionary perspective but not yet informative. There are too few real data points $(n=7$ pathogens) and a better study for evolutionary questions might centre on a single tick population maintaining several pathogens, or the same pathogen existing in several geographically distinct tick populations. We also note here that De Matos \& Matos (1998) discovered that properties of projection matrices, which reflect the biology of the species modelled, can give rise to relationships between the element elasticities. This can in turn create artificial constraint 'lines' in these ternary plots as to where the balance of contributions can actually fall. In Fig. 2, the clouds of points from the Latin Hypercube sampling of parameter ranges reveal a vague line running across the ternary plot that divides the three tick-borne pathogens closest to the upper corner from the other four. The concern then is the ability to separate new information about a set of pathogens (or plants or animals) from the already well-known biology that is reflected in the matrices themselves.

In the same way that elasticities of projection matrices have informed conservation efforts, such as for the Serengeti cheetah population (Acinonyx jubatus) and Kemp's ridley sea turtles (Lepidochelys kempir; Benton \& Grant 1999), elasticities of NGMs may inform the theory and practice of disease control. That is, the elasticity value for a transmission route, or a particular element of the NGM, suggests that control methods affecting that route or element will have the most effective impact on $R_{0}$. For example, our results for Lyme disease provide broad theoretical support for the suggestion to vaccinate wild mouse reservoirs as an intervention strategy (Tsao et al. 2004) as $R_{0}$ is predicted to drop rapidly if systemic transmission is reduced. However, two qualifications must be raised at this point, both of which have arisen in the context of the equivalent use of elasticity analysis in evolutionary and population ecology (Benton \& Grant 1999). First, an NGM represents a linearization of the system when all hosts of all host types are susceptible. That is, an NGM makes no attempt to model the disease dynamics beyond the initial growth in infected as it is wholly a means of calculating $R_{0}$. This is equivalent to the concern that projection matrices do not take into account density dependence that will arise from resource limitations as a population grows. For example, the endemic equilibrium for a pathogen is determined by a very different set of factors as to those that determine $R_{0}$, such as the rate at which new susceptibles enter the population. The aim of control measures will be context-dependent, and vary from lowering equilibrium prevalence to preventing diseases from expanding into new areas or eliminating disease. Elasticity analyses of NGMs, because the focus is on $R_{0}$, are relevant to the latter but not the former. The second qualification is that the sensitivities and elasticities of $R_{0}$ to elements of the parameters are local properties of the matrix. That is, they are evaluated at a particular point in parameter space by varying just one parameter and holding all others constant. We have partially overcome this problem here by replacing point estimates by ranges for parameter values (creating a parameter space which we sampled using Latin Hypercube sampling), but we note here the availability of methods such as Sobol's (1993), which can take into account interactions between parameters. For matrix models in general though, this method and other global sensitivity approaches (Saltelli et al. 2000) would move away from the central advantage of composite elasticities, which is that because they are built from the simpler element elasticities that sum to 1 , they have an interpretation as contributions.

Whether NGMs will play a broader role in understanding the ecology of infectious diseases, as projection matrices did for the population biology of plants and animals remains to be seen. In the context of tick-borne pathogens at least, the methodology is a promising approach to comparing strains of the same pathogen or pathogens co-existing in the same tick population. However, there is a much larger group of multi-host pathogens of public health or veterinary importance for which the methodology presented here stands to improve the scientific basis for their management. Such a group would include the true multi-host pathogens such as the rabies virus, Echinococcus multilocularis, plague, rinderpest, Mycobacterium bovis and the African trypanosomes. On this basis, we see perturbation analyses of NGMs as a much needed tool to meet the scientific challenges posed by the complex ecology of multi-host pathogens.

\section{ACKNOWLEDGEMENTS}

This publication has been funded under the EU's 6th Framework Program for Research, Technological Development and Demonstration, Priority 1.1.6.3 Global Change and Ecosystems (European Commission, DG Research, 
GOCECT-2003-010284 EDEN) and is officially catalogued by the EDEN Steering Committee as EDEN0141. The content of this article does not represent the official position of the European Commission and is entirely under the responsibility of the authors. The work of Stephen Davis and Hans Heesterbeek was supported by the Netherlands Organisation for Scientific Research (NWO/ZonMw grant 918.56.620). The authors thank Marieke Jesse for helpful discussions on elasticity analysis. They also thank Sarah Randolph and Durland Fish for sharing their expertise on tick-borne disease ecology and epidemiology.

\section{REFERENCES}

Benton, T.G. \& Grant, A. (1999). Elasticity analysis as an important tool in evolutionary and population ecology. Trends Ecol. Evol., 14, 467-471.

Brault, S. \& Caswell, H. (1993). Pod-specific demography of killer whales (Orcinus orca). Ecology, 74, 1444-1454.

Burgdorfer, W. \& Brinton, L.P. (1975). Mechanisms of transovarial infection of spotted fever rickettsiae in ticks. Ann. NY Acad. Sci., 266, 61-72.

Burgdorfer, W. \& Varma, G.R. (1967). Trans-stadial and transovarial development of disease agents in arthropods. Annu. Rev. Entomol., 12, 347-376.

Caswell, H. (2001). Matrix Population Models: Construction, Analysis, and Interpretation, 2nd edn. Sinauer Associates Sunderland, MA, USA.

De Matos, M.B. \& Matos, D.M.S. (1998). Mathematical constraints on transition matrix elasticity analysis. J. Ecol., 86, 706-708.

Diekmann, O. \& Heesterbeek, J.A.P. (2000). Mathematical Epidemiology of Infectious Diseases: Model Building, Analysis and Interpretation. John Wiley \& Sons, Ltd., New York.

Diekmann, O., Heesterbeek, J.A.P. \& Metz, J.A.J. (1990). On the definition and the computation of the basic reproduction ratio $R_{0}$ in models for infectious diseases in heterogeneous populations. J. Math. Biol., 28, 365-382.

Gandon, S., Mackinnon, M.J., Nee, S. \& Read, A.F. (2001). Imperfect vaccines and the evolution of pathogen virulence. Nature, 414, 751-755.

Hartemink, N.A., Randolph, S.E., Davis, S.A. \& Heesterbeek, J.A.P. (2008). The basic reproduction number for complex disease systems: defining $R_{0}$ for tick-borne infections. Am. Nat., 171, 743-754.

de Kroon, H., Plaisier, J.A., van Groenendael, J. \& Caswell, H. (1986). Elasticity: the relative contribution of demographic parameters to population growth rate. Ecology, 67, 1427-1431.

Macaluso, K.R., Sonenshine, D.E., Ceraul, S.M. \& Azad, A.F. (2001). Infection and transovarial transmission of Rickettsiae in Dermacentor variabilis ticks acquired by artificial feeding. Vector Borne Zoonotic Dis., 1, 45-53.

Randolph, S.E. (2000). The shifting landscape of tick-borne zoonoses: tick-borne encephalitis and Lyme borreliosis in Europe. Philos. Trans. R. Soc. Lond. B Biol. Sci., 356, 1045-1056.

Randolph, S.E., Miklisova, D., Lysy, J., Rogers, D.J. \& Labuda, M. (1999). Incidence from coincidence: patterns of tick infestations on rodents facilitate transmission of tick-borne encephalitis virus. Parasitology, 118 (Pt 2), 177-186.
Roberts, M.G. \& Heesterbeek, J.A. (2003). A new method for estimating the effort required to control an infectious disease. Proc Biol Sci., 270, 1359-64.

Saltelli, A., Chan, K. \& Scott, E.M. (2000). Sensitivity Analysis. Series in Probability and Statistics. Wiley, Chichester, West-Sussex, England.

Sanchez, M.A. \& Blower, S.M. (1997). Uncertainty and sensitivity analysis of the basic reproductive rate: tuberculosis as an example. Am. J. Epidemiol., 145, 1127-1137.

Silvertown, J., Franco, M. \& Menges, E. (1996). Interpretation of elasticity matrices as an aid to the management of plant populations for conservation. Conserv. Biol., 10, 591-597.

Sobol, I.M. (1993). Sensitivity analysis for non-linear mathematical models. Math. Model. Comput. Exp., 1, 407-414.

Steere, A.C. (2001). Lyme disease. N. Engl. J. Med., 345, 115-125.

Tsao, J.I., Wootton, J.T., Bunikis, J., Luna, M.G., Fish, D. \& Barbour, A.G. (2004). An ecological approach to preventing human infection: Vaccinating wild mouse reservoirs intervenes in the Lyme disease cycle 10.1073/pnas.0405763102. PNAS 101, 18159-18164.

Van Groenendael, J., de Kroon, H. \& Caswell, H. (1988). Projection matrices in population biology. Trends Ecol. Evol., 3, 264-269.

Woolhouse, M.E.J., Tailor, L.H. \& Haydon, D.T. (2001). Population Biology of Multihost Pathogens. Science, 292, 1109-1112.

\section{SUPPORTING INFORMATION}

Additional Supporting Information may be found in the online version of this article:

Figure $\mathrm{S} 1$ shows the association between $\mathrm{R}_{0}$ and the dominant transmission routes; the contribution to $R_{0}$ of the transmission route with the highest contribution to $R_{0}$ (i.e. the dominant route) is plotted against $R_{0}$ for 1000 samples per pathogen.

Figure S2 shows all the contributions of transmission routes plotted separately against $R_{0}$ for all seven pathogens.

Tables S1 and S2 provide the values of tick and pathogenrelated parameters, respectively.

Appendix S1 Additional information on materials and methods. (i) Description of the next-generation matrix, (ii) Additional information on the parameter value estimate and (iii) Latin Hypercube sampling and elasticity analysis.

As a service to our authors and readers, this journal provides supporting information supplied by the authors. Such materials are peer-reviewed and may be re-organized for online delivery, but are not copy-edited or typeset. Technical support issues arising from supporting information (other than missing files) should be addressed to the authors.

Editor, Giulio De Leo

Manuscript received 23 June 2009

First decision made 2 August 2009

Manuscript accepted 7 August 2009 